\title{
A ação da identidade social sobre o comportamento discricionário de funcionários: uma perspectiva brasileira
}

\author{
The action of social identity on the discretionary \\ behavior of employees: a Brazilian perspective
}

\author{
Rogerio Scabim Morano ${ }^{1}$ \\ Alcides Barrichello² \\ Rafael Ricardo Jacomossi ${ }^{3}$
}

\section{Resumo}

Os estudos acerca da temática sobre identidade social nas organizações e seus efeitos sobre o comportamento discricionário de funcionários são pouco explorados no Brasil. Por outro lado, pesquisas versam sobre a influência de recompensas econômicas e procedimentos justos como influenciadores desse tipo de comportamento. Estudo realizado nos Estados Unidos verificou o grau de mediação da variável identidade social entre esses elementos. A partir deste estudo, realizouse em empresas brasileiras uma pesquisa utilizando a técnica de modelagem por equações estruturais, cujos resultados, diferentemente do trabalho americano, indicaram que o efeito de recompensas econômicas e procedimentos justos sobre o comportamento discricionário só se verifica por meio da mediação da identidade social. Do ponto de vista gerencial, esses resultados trazem uma significante contribuição no sentido de reforçar a importância da variável identidade social como influenciadora do comportamento discricionário de funcionários.

Palavras-chave: Identidade social no trabalho. Comportamento discricionário. Modelo de equações estruturais.

Professor Doutor do Departamento de Ciências Exatas e da Terra da Universidade Federal de São Paulo - UNIFESP - Brasil - E-mail: r.morano@uol.com.br

2 Doutorando do Centro Universitário da FEl, Professor de Pós-Graduação Lato Sensu da Universidade Presbiteriana Mackenzie - Brasil - E-mail: alcidesbarrichel@uol.com.br

3 Professor Doutor do Departamento de Administração do Centro Universitário da FEI - Brasil E-mail: rafaeljacomossi@gmail.com 


\section{Abstract}

It has been little explored in Brazil studies on the theme of social identity in organizations and their effect on the extrarole behavior of employees. On the other hand, researches reveal the influence of economic outcomes and procedural justice as influencers of this type of behavior. A study conducted in the United States found the mediation of the variable social identity among these elements. From this study, it was carried out research in Brazilian companies using structural equation modeling technique (SEM), such results, unlike the American work, indicated that the effect of economic outcomes and procedural justice on the extrarole behavior is verified only through the mediation of social identity. About a managerial point of view, these results provide a significant contribution to reinforce the importance of the social identity as influencer on the extrarole behavior of employees.

Keywords: Social identity at work. Extrarolebehavior.Structuralequationmodeling

\section{Introdução}

No campo da administração, poucas são as pesquisas que relacionam o comportamento discricionário de grupos de trabalhos e os efeitos sobre o desempenho das firmas, destacando-se os trabalhos de Bakker et al. (2011), Kataria et al. (2013) e Kennedy e Daim (2010). Também em número reduzido são os trabalhos que relacionam o comportamento discricionário como decorrente da identidade social (BLADER; TYLER, 2009; HASLAM, 2004; TYLER; BLADER, 2000), constituindo-se essa temática, portanto, em terreno fértil para que pesquisas possam ser desenvolvidas, e com isso, avançar na discussão.

A definição de identidade social nesse contexto se caracteriza pelo sentimento de pertencimento que um indivíduo ou um grupo de indivíduos sente em relação a uma organização. Integrar a teoria de identidade social às organizações fornece subsídios de como a pesquisa em psicologia pode ajudar a entender a dinâmica entre pessoas e qual o efeito desta no contexto organizacional (BLADER; TYLER, 2009). Haslam (2004) reforça o papel dessa teoria como elemento fundamental para se compreender a vida organizacional.

Com essa perspectiva, Blader e Tyler (2009) realizaram pesquisa com funcionários de uma empresa do ramo financeiro nos 
Estados Unidos na qual testaram o nível de engajamento do grupo de trabalho, representada pelo comportamento discricionário, como sendo decorrente da percepção das variáveis procedimentos justos e recompensas econômicas, havendo uma ação mediadora feita pela variável identidade social. A variável "comportamento discricionário" é definida como a representação das funções ou atividades exercidas pelo funcionário que estão além daquelas constantes em seu contrato de trabalho (PODSAKOFF et al., 2000). Procedimentos justos indicam o nível de satisfação que um funcionário apresenta em relação ao tratamento que lhe é dispensado na organização (TYLER; BLADER, 2000). Com relação à variável "recompensas econômicas", ela se situa na esfera do nível de satisfação dos funcionários com o valor financeiro que recebem por meio de salários e/ou programa de bônus, sendo esse fator explorado há bastante tempo na literatura como sendo um importante motivador (ADAMS, 1965; BLAU, 1964; HOMANS, 1961; THIBAUT; KELLEY, 1959).

No Brasil, essas temáticas foram estudadas separadamente (ASSMAR et al., 2005; VASCONCELOS; VASCONCELOS, 2002), não tendo havido estudos similares aos de Blader e Tyler (2009), que agregam as variáveis procedimentos justos, recompensas econômicas, identidade social e comportamento discricionário. Dessa forma, suscitase o seguinte problema de pesquisa: as variáveis "comportamento justo" e "recompensas econômicas" têm sua ação sobre o comportamento discricionário de grupos de trabalhos em empresas brasileiras, sendo essas mediadas pela identidade social? O principal objetivo deste estudo, por meio da percepção de funcionários, é verificar o papel mediador da variável identidade social, sendo, então, caracterizada pela seguinte hipótese: a identidade social é mediadora entre recompensas econômicas e procedimentos justos e a variável "comportamento discricionário" em empresas brasileiras.

Outra contribuição desta pesquisa reside em trazer para o campo dos estudos organizacionais as temáticas da identidade social e comportamento discricionário, investigadas pelo método de equações estruturais, e a partir dela, motivar novos estudos com base nessa 
perspectiva. Complementarmente, como contribuição gerencial, fica clara a necessidade de se construir nas empresas a identidade social como um fator necessário para o desenvolvimento do comportamento discricionário dos funcionários, interesse esse das organizações que buscam alcançar melhores resultados (DUBINSKY; SKINNER, 2002).

\section{Referencial teórico}

\subsection{Procedimentos justos}

Pesquisas têm demonstrado que a justiça é a primeira dimensão que as pessoas usam para avaliar os processos e tratamentos que estas encontram nos grupos (TYLER, 2000; TYLER; BLADER, 2000). Isso é refletido nos seus julgamentos acerca de quão envolvidas essas pessoas se sentem nesses grupos. Há uma série de fatores que os funcionários utilizam para avaliar a qualidade e a justiça do tratamento, como decisões sobre política de remuneração e promoção, forma de tratamento entre superiores e subordinados, dentre outros.

De forma ampla, a justiça organizacional deve ser entendida como sendo a percepção de justiça pelos funcionários no que tange às relações de trabalho na organização (ASSMAR et al., 2005).

Procedimentos justos (procedimentos de tomada de decisão percebidos pelos funcionários como justos) contribuem para uma sensação de conexão social com o coletivo, enquanto procedimentos injustos (procedimentos de tomada de decisão percebidos como injustos) projetam um sentimento de exclusão. Assim, procedimentos injustos causam prejuízo à cooperação dos funcionários porque dificultam a conexão social entre os funcionários (VAN DIJKE et al., 2015).

Birnberg et al. (2008) corroboraram com o que foi mencionado, afirmando que se o funcionário acredita que sua relação de resultado versus trabalho é injusta quando comparada a de outros, ele irá sentir emoções negativas. Ao tentar minimizá-las, aumentará ou diminuirá seu empenho e/ou resultados, dependendo do que for mais apropriado na 
ocasião. Se, por exemplo, pessoas de vendas percebem que não estão sendo recompensadas justamente pelos resultados que estão gerando, elas podem tomar medidas unilaterais para corrigir este desequilíbrio. Tais ações podem incluir o distanciamento psicológico do trabalho, redução do esforço ou mesmo abandono da empresa. Nenhuma dessas alternativas corrige a percepção do funcionário sobre a injustiça, nem qualquer um deles levará alguém a manter ou aumentar seu esforço no trabalho, que em última análise é o que interessa à empresa (DUBINSKY; SKINNER, 2002).

Com efeito, os funcionários não são susceptíveis a assumir trabalho adicional, seja designado pela administração ou não. Netemeyer et al. (1997) mostraram uma associação positiva entre a percepção de justiça na designação de recompensas econômicas e comportamento discricionário de vendedores. Já Dubinsky e Skinner (2002) indicaram que indivíduos entram em um trabalho com certos ativos (por exemplo, educação, treinamento, habilidade, experiência) e recebem algumas recompensas, extrínsecas e/ou intrínsecas. A injustiça pode ter um impacto adverso sobre o retorno percebido relacionado ao trabalho, como motivação e satisfação.

\subsection{Recompensas econômicas}

Os sistemas de recompensa podem prover vantagem competitiva às organizações. Nesse sentido, o sistema de pagamentos dos funcionários interfere no desempenho da firma, podendo ser planejado estrategicamente e se relacionando às atividades, atributos e resultados de trabalhos que permitem que se atinjam os objetivos planejados (HOWARD; DOGHERTY, 2011).

Por outro lado, estudos demonstram que nem sempre o comportamento discricionário dos funcionários é conseguido mediante altas remunerações. Howard e Dogherty(2011) revelaram que embora estratégias de compensação proponham que recompensas baseadas em resultados encorajem tal comportamento dos funcionários, essa se aplica mais para aqueles que exercem trabalhos manuais ou 
operacionais. Contudo, os autores confirmam que, de forma geral, os funcionários associam o trabalho que realizam com as recompensas financeiras que recebem.

Já Leea et al. (2011) examinaram as preferências de pagamentos comparando salários baseados em desempenho e por senioridade, seus antecessores e consequentes em termos de formação da cultura organizacional. Os resultados indicaram preferência pelo sistema de pagamento baseado em desempenho, sobretudo para aqueles funcionários em início de carreira. Todavia, não foi observada relação desta com a formação da cultura organizacional.

Dessa forma, o comportamento discricionário do grupo de trabalho pode ou não ser influenciado diretamente pela percepção de que tão alta ou não é a recompensa econômica obtida pelos funcionários (HAWORTH; LEVY, 2001; PODSAKOFF et al., 2000). Isso demonstra que nem sempre os funcionários agem nas suas empresas baseados em interesse em pagamentos e benefícios (GINTIS et al., 2005; HASLAM; ELLEMERS, 2005). Contudo, recompensas econômicas favoráveis comunicam uma mensagem positiva para os empregados sobre essas questões, enquanto recompensas econômicas negativas comunicam uma mensagem truncada. Para fornecer uma resposta, Tyler e Blader (2000, 2003) construíram um modelo de comportamento discricionário do grupo em que os valores simbólicos das recompensas econômicas são relevantes para reforçar a identidade social dos indivíduos, e este sentimento denota atitudes positivas que são disseminadas por meio de comportamentos além dos estipulados no contrato de trabalho (comportamento discricionário). Os autores partem do pressuposto de que os funcionários fazem julgamentos diferenciados dos pagamentos e benefícios que recebem da empresa quando estes mesmos estão inseridos como membros integrantes de um grupo.

\subsection{Identidade social}

A definição de identidade social se caracteriza pelo sentimento de pertencimento e continuidade que uma pessoa ou um grupo de 
pessoas sente em relação a uma organização (BLADER; TYLER, 2009). Refere-se ao esforço da pessoa em realizar uma síntese de sua ação, equilibrando as forças internas e as externas que influenciam tal ação, fruto do inter-relacionamento de sua realidade interior e a realidade externa construída pelo grupo social. O reconhecimento dos outros é um dos elementos fundamentais na construção da identidade da pessoa (VASCONCELOS; VASCONCELOS, 2002).

O sentido de identidade no contexto do trabalho, dessa forma, depende de jogos de identificação e participação, de forma que os funcionários em geral esperam de seu trabalho uma retribuição moral dada através do reconhecimento. Tal reconhecimento de utilidade, conferido pela hierarquia, pelos pares ou pelo grupo social, influi na percepção que os funcionários desenvolvem sobre o valor social de seu trabalho e no conceito que desenvolvem de si próprios (DEJOURS, 1996).

Konovsky e Pugh (1994) afirmaram que a identidade social desenvolvida nas organizações implica diretamente no comportamento de grupo apresentado pelos membros da organização. Esse comportamento pode ser considerado um tipo particular de favoritismo em grupo e é definido como o comportamento do empregado que está acima e além de seu dever formalmente explicito e pode não ser recompensados no contexto de uma organização formal.

A identidade social é compartilhada por aqueles que ocupam posições semelhantes e os levam a perceber a semelhança. Por outro lado, leva também à percepção de diferenças em relação aos membros de outros grupos ou categorias. Nas interações sociais, desenvolvendose ao longo do tempo, é que a identidade se constrói ou evolui (DECHAMPS; MOLINER, 2009).

Processos psicológicos tendem a organizar o entorno em termos de categorias: grupos de pessoas, de objetos, de eventos, enquanto semelhantes para determinadas ações, intenções ou atitudes. Esse processo de categorização reagrupa os objetos que são ou que parecem ser semelhantes uns aos outros em certas dimensões. A 
categorização encontra então sua função no papel que ela desempenha na sistematização do entorno, sua construção e organização (TAJFEL, 1982).

\subsection{Comportamento discricionário}

Kataria et al. (2013) sugeriram que os valores base da organização por meio de uma arquitetura de Recursos Humanos sustentam níveis altos de engajamento dos funcionários para que a organização alcance efetividade. Empregados engajados se envolvem nas suas experiências de trabalho de forma estimulante e com concentração, gerando consequências positivas para as organizações (BAKKER et al., 2011; BAKKER; BAL, 2010). Adicionalmente, empregados que experimentam grande paixão pelo seu trabalho, produzem bens de melhor qualidade e sentem que a sua contribuição ajuda a mover a empresa para adiante (KENNEDY; DAIM, 2010).

Ao tratar o tema "engajamento dos funcionários em seu trabalho", Bladere Tyler (2009) sugeriram que este pode ser caracterizado por tarefas exercidas além de seu contrato de trabalho, formando o comportamento discricionário. O comportamento discricionário, por sua vez, é uma ação individual e voluntária do funcionário, não coordenada ou dirigida por seu gerente ou supervisor, dependendo única e exclusivamente de sua vontade e do desejo de se atingir determinado resultado. Ele é caracterizado pelo desempenho (ou atividades), em que o funcionário vai além de seu dever, excedendo as demandas normais, exigências ou expectativas de seu trabalho (DUBINSKY; SKINNER, 2002).

Blader e Tyler (2009) mostraram em seu trabalho importantes vínculos entre identidade social e o comportamento discricionário dos empregados, indicando que a identidade social que os funcionários formam em torno da organização na qual trabalham está fortemente relacionada ao comportamento discricionário demonstrado em relação a temas ligados à empresa. 


\section{Metodologia}

A coleta de dados foi realizada por meio de questionário eletrônico preenchido pela web, empregando-se a ferramenta Google Docs ${ }^{\circledR}$, no período de abril a maio de 2015. A ferramenta foi disponibilizada pelas redes sociais Facebook ${ }^{\circledast}$ e Linkedln $n^{\circledast}$. Adicionalmente às escalas, foram inseridas seis questões para qualificação do perfil dos respondentes que participaram da pesquisa como pode ser verificado nas tabelas 1 e 2 . Foram obtidas 221 respostas, sem dados faltantes. Todos os cálculos foram realizados por meio dos softwares IBM SPSS Statistics ${ }^{\circledR} 20.0$ (análise descritiva e análise fatorial exploratória) e IBM SPSS Amos ${ }^{\circledR}$ 22.0 (modelagem por equações estruturais).

Observando-se os dados das Tabelas 1 e 2, é possível verificar que existe certo equilíbrio entre os gêneros dos respondentes, mais de $60 \%$ são jovens com até 25 anos, mais de $20 \%$ ocupam cargos de gestão, havendo certa distribuição igualitária entre suas áreas de atuação. Um terço das empresas possui até 100 funcionários, e um quarto delas acima de 5.000. Vale destacar que por volta de $40 \%$ delas, atuam nas áreas de educação e pesquisa, prestação de serviços, química e produtos de consumo. É possível verificar a existência de certa homogeneidade no que diz respeito ao gênero, área de atuação do respondente, tipo de indústria e porte das empresas abrangidas, aumentando a representatividade da amostra e consequente significância dos resultados obtidos.

O questionário foi desenvolvido com base na pesquisa de Blader e Tyler, (2009), utilizando-se das mesmas escalas propostas por esses autores que constou de afirmações a respeito de Procedimentos Justos (ProJus), Identidade Social/Identificação (Soidid), Identidade Social/ Orgulho (Soidpri), Identidade Social/Respeito (Soidres), Recompensas Econômicas/Remuneração (Ouevpay), Recompensas Econômicas/ Expectativas Futuras (Ouevfut), Recompensas Econômicas/ Oportunidades Justas (Ouevfai) e Comportamento Discricionário (Exrobe). 
Tabela 1: Perfil dos respondentes

\begin{tabular}{|c|c|c|}
\hline GÊNERO & FREQUÊNCLA & PORCENTAGEM \\
\hline Feminino & 101 & $45,7 \%$ \\
\hline Masculino & 120 & $54,3 \%$ \\
\hline IDADE & FREQUÊNCIA & PORCENTAGEM \\
\hline Entre 17 e 25 anos & 139 & $62,9 \%$ \\
\hline Entre 26 e 35 anos & 49 & $22,2 \%$ \\
\hline Entre 36 e 45 anos & 19 & $8,6 \%$ \\
\hline Acima de 45 anos & 14 & $6,3 \%$ \\
\hline CARGO NA EMPRESA & FREQUÊNCIA & PORCENTAGEM \\
\hline Assistente & 43 & $19,5 \%$ \\
\hline Supervisor/Coordenador & 23 & $10,4 \%$ \\
\hline Analista & 20 & $9,0 \%$ \\
\hline Gerente & 14 & $6,3 \%$ \\
\hline Diretor & 11 & $5,0 \%$ \\
\hline Outros & 110 & $49,8 \%$ \\
\hline ÁREA DE ATUAÇÃO & FREQUÊNCIA & PORCENTAGEM \\
\hline Vendas & 32 & $14,5 \%$ \\
\hline Marketing & 19 & $8,6 \%$ \\
\hline Produção & 19 & $8,6 \%$ \\
\hline Planejamento & 14 & $6,3 \%$ \\
\hline Logística & 11 & $5,0 \%$ \\
\hline Recursos humanos & 11 & $5,0 \%$ \\
\hline Finanças & 9 & $4,1 \%$ \\
\hline Controle de qualidade & 8 & $3,6 \%$ \\
\hline TI & 6 & $2,7 \%$ \\
\hline Compras & 4 & $1,8 \%$ \\
\hline Auditoria & 3 & $1,4 \%$ \\
\hline Outros & 85 & $38,5 \%$ \\
\hline
\end{tabular}

Fonte: Elaborada pelos autores.

A tradução das afirmações da língua inglesa para a portuguesa foi submetida a cinco especialistas, sendo três doutores da área de Administração e dois profissionais seniores do mercado. A versão final em português do questionário foi submetida a um pré-teste com dez pessoas para avaliação do tempo a ser gasto no preenchimento das respostas, facilidade de entendimento das frases utilizadas, proposição de reconstrução de frases ou ordenação de palavras. A ferramenta de coleta foi então enviada aos respondentes, após a conciliação das avaliações e sugestões de alteração dos avaliadores do pré-teste. As 
dimensões foram medidas por meio de escala Likert, variando de 1 a 7 (VIEIRA; DALMORO, 2008), na qual o respondente preencheu seu nível de opinião com relação às diferentes afirmações variando de "discordo totalmente" a "concordo totalmente", "de modo algum" a "muitíssimo", "nunca" a "sempre" e "muito desfavorável" a "muito favorável".

Tabela 2: Perfil das empresas dos respondentes

\begin{tabular}{|c|c|c|}
\hline TIPO DE INDUSTRIA & FREQUENNCIA & PORCENTAGEM \\
\hline Química & 33 & $14,9 \%$ \\
\hline Prestação de serviços & 30 & $13,6 \%$ \\
\hline Educação e pesquisa & 19 & $8,6 \%$ \\
\hline Financeiro & 15 & $6,8 \%$ \\
\hline Produtos de consumo & 14 & $6,3 \%$ \\
\hline Setor público & 9 & $4,1 \%$ \\
\hline Beleza e saúde & 8 & $3,6 \%$ \\
\hline Ciências da vida & 8 & $3,6 \%$ \\
\hline Varejo & 8 & $3,6 \%$ \\
\hline Alta tecnologia & 5 & $2,3 \%$ \\
\hline Automobilistica & 4 & $1,8 \%$ \\
\hline Esporte e entretenimento & 4 & $1,8 \%$ \\
\hline Seguros & 4 & $1,8 \%$ \\
\hline Telecomunicações & 4 & $1,8 \%$ \\
\hline Óleo e gás & 3 & $1,4 \%$ \\
\hline Engenharia e construção & 2 & $0,9 \%$ \\
\hline Transporte e logistica & 2 & $0,9 \%$ \\
\hline Energia & 1 & $0,5 \%$ \\
\hline Máquinas e componentes & 1 & $0,5 \%$ \\
\hline Papel e celul ose & 1 & $0,5 \%$ \\
\hline Utilidades & 1 & $0,5 \%$ \\
\hline Outros & 45 & $20,4 \%$ \\
\hline NÚMERO DE F UNCIONÁRIOS & FREQUENCIA & PORCENTAGEM \\
\hline 1 a 100 & 74 & $33,5 \%$ \\
\hline 101 a 500 & 33 & $14,9 \%$ \\
\hline 501 a 1000 & 27 & $12,2 \%$ \\
\hline 1001 a 5000 & 32 & $14,5 \%$ \\
\hline Acima de 5000 & 55 & $24,9 \%$ \\
\hline
\end{tabular}

Fonte: Elaborada pelos autores.

O modelo conceitual, baseado em Blader e Tyler, (2009), relaciona Procedimentos Justos e Recompensas Econômicas com Comportamentos Discricionários, tendo a Identidade Social como possível mediadora dessas relações, como se observa na Figura 1. 
Figura 1: Modelo conceitual

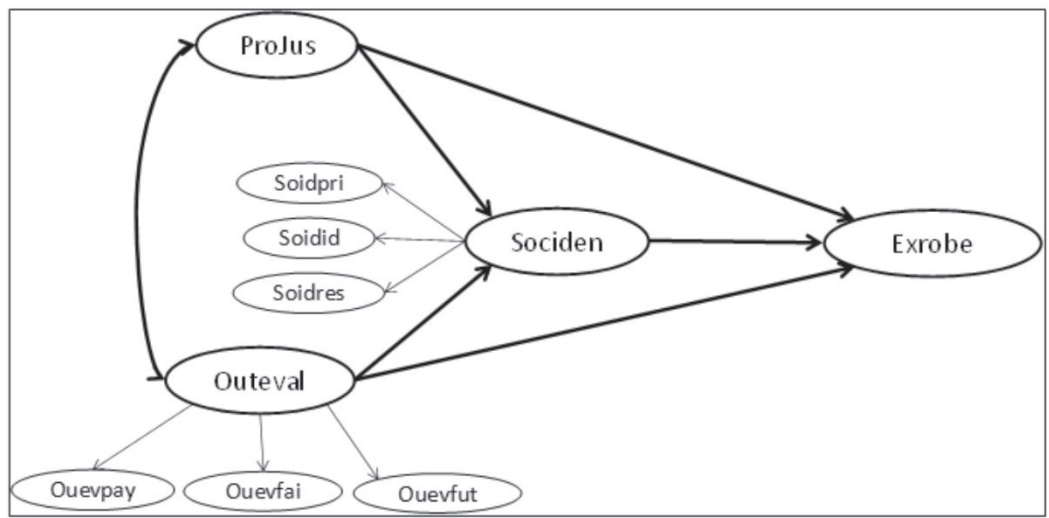

Fonte: Baseado em Blader e Tyler (2009)

Uma variável mediadora, representada acima por Sociden, é aquela que age como interveniente entre duas variáveis numa relação, ou seja, ela recebe influência da variável independente e influencia a variável dependente (BARON; KENNY, 1986). Como pode ser visto na Figura 1, os construtos Soidpri, Soidid e Soidres formam o construto de segunda ordem Identidade Social (Sociden) (BYRNE, 2009), assim como Ouevpay, Ouevfut e Ouevfai formam o construto de segunda ordem Recompensas econômicas (Outeval).

\section{Análise e discussão dos resultados}

Depois de realizada a coleta dos dados, procedeu-se a análise fatorial exploratória, conforme as Tabelas 3 e 4. 
Tabela 3: Analise fatorial exploratória (primeira parte)

\begin{tabular}{|c|c|c|c|c|}
\hline SIGLA & CONSTRUCTO & IIEM & $\begin{array}{l}\text { CARGA } \\
\text { FATORIAL }\end{array}$ & ALFA \\
\hline \multirow[t]{9}{*}{ ProJus } & \multirow[t]{9}{*}{ Procedimentos Justos } & $\begin{array}{l}\text { 1. As decisões que me afetam geralmente são tomadas de forma justa na organização } \\
\text { onde trabalho. }\end{array}$ & 0,894 & \multirow[t]{9}{*}{0,959} \\
\hline & & 2. Minha organização se empenha em tomar decisões que me afetem de forma justa. & 0,864 & \\
\hline & & $\begin{array}{l}3 \text { As decisões são tomadas geralmente de forma justa na organização onde eu } \\
\text { trabalho. }\end{array}$ & 0,924 & \\
\hline & & 4. Minha organização se esforça para tomar decisões de uma forma justa. & 0,907 & \\
\hline & & $\begin{array}{l}\text { 5. A maioria das questões em que estou envolvido são resolvidos de maneira justa } \\
\text { onde trabalho }\end{array}$ & 0,830 & \\
\hline & & 6. No geral, sou tratado comj ustiça onde trabalho & 0,869 & \\
\hline & & 8. No geral, as pessoas são tratadas com justiça onde trabalho. & 0,856 & \\
\hline & & $\begin{array}{l}\text { 9. Os critérios usados nas decisões sobre pagamento e promoções são justas onde } \\
\text { trabalho }\end{array}$ & 0,739 & \\
\hline & & $\begin{array}{l}\text { 10. Há um sentimento geral entre os funcionários de que as coisas são tratadas de } \\
\text { forma justa onde eu trabal ho. }\end{array}$ & 0,844 & \\
\hline \multirow[t]{5}{*}{ Soidid } & \multirow[t]{5}{*}{$\begin{array}{l}\text { Identidade Social - } \\
\text { Identificação }\end{array}$} & $\begin{array}{l}\text { 1. Trabalhar na minha organização é importante para a maneira como me vejo como } \\
\text { pessoa. }\end{array}$ & 0,849 & \multirow[t]{5}{*}{0,854} \\
\hline & & $\begin{array}{l}\text { 2. Quando alguém elogia as realizações da organização onde trabalho, sinto como se } \\
\text { fosse um elogio para mim. }\end{array}$ & 0,748 & \\
\hline & & $\begin{array}{l}\text { 3. Quando alguem de fora critica a organização onde trabalho, sinto isso como se } \\
\text { fosse uma ofensa pessoal. }\end{array}$ & 0,746 & \\
\hline & & 4. O lugar em que eu trabalho diz muito sobre quem eu sou como pessoa. & 0,870 & \\
\hline & & 5. Penso que sou parecido com as pessoas que trabalham na minha organização. & 0,763 & \\
\hline \multirow[t]{5}{*}{ Soidpri } & \multirow{5}{*}{$\begin{array}{l}\text { Identidade Social - } \\
\text { Orgulho }\end{array}$} & 1. Minha organizaçăo é uma das melhores do seu ramo. & 0,852 & \multirow[t]{5}{*}{0,857} \\
\hline & & 2. As pessoas ficam impressionadas quando digo onde eu trabalho. & 0,703 & \\
\hline & & 3. Minha organização é bem respeitada em seu ramo. & 0,805 & \\
\hline & & 4. Considero que onde trabalho tem um efeito positivo sobre mim. & 0,772 & \\
\hline & & 5. Sinto orgulho de dizer aos outros onde trabal ho. & 0,865 & \\
\hline \multirow[t]{7}{*}{ Soidres } & \multirow{7}{*}{$\begin{array}{l}\text { Identidade Social - } \\
\text { Respeito }\end{array}$} & Aval ie o quanto seus ge stores ... & & \multirow[t]{7}{*}{0,899} \\
\hline & & 1.... respeitam o trabalho que você faz & 0,810 & \\
\hline & & 2.... respeitam suas ideias relacionadas com o trabalho. & 0,864 & \\
\hline & & 3....acreditam na qualidade do seu trabalho. & 0,866 & \\
\hline & & 4. ...consideram suas contribuições ao trabal ho únicas. & 0,849 & \\
\hline & & 5. ...pensam que você tem insights e ideias valiosas. & 0,828 & \\
\hline & & 6. pensam que seria dificil substituir voce. & 0,700 & \\
\hline
\end{tabular}

Fonte: Elaborada pelos autores.

Os resultados apresentados nas Tabelas 3 e 4 foram calculados e analisados conjuntamente. A quebra em duas tabelas foi realizada apenas para facilitar a apresentação e formatação dos dados.

Foram analisadas as cargas fatoriais de cada uma das afirmações propostas para verificação de suas significâncias e da representatividade da amostra utilizada. De acordo com HAIRet al. (2009), cargas fatoriais maiores que 0,40 garantem significância de amostras superiores a 200 registros. Conforme apresentam as Tabelas 3 e 4, todas as cargas encontradas para as respostas são superiores a esse valor mínimo, o que valida o tamanho da amostra de 221 respondentes. 
Tabela 4: Analise fatorial exploratória (segunda parte)

\begin{tabular}{|c|c|c|c|c|}
\hline SIGLA & CONSTRUCTO & ITEM & $\begin{array}{l}\text { CARGA } \\
\text { FATORIAL }\end{array}$ & ALFA \\
\hline \multirow[t]{5}{*}{ Ouevpay } & Recompensas & 1. Quão favorável é remuneração que você recebe na organização onde trabal ha? & 0,954 & 0,946 \\
\hline & Econômicas - & 2. Quão favorável são os beneficios que você recebe na organização onde trabalha? & 0,766 & \\
\hline & Remuneração & $\begin{array}{l}\text { 3. Comparado com suas expectativas, quão favorável é sua remuneração pelo trabalho } \\
\text { que você realiza? }\end{array}$ & 0,901 & \\
\hline & & 4. Quão favorável é o seu salário? & 0,953 & \\
\hline & & 5. Quão favorável é a remuneração que você re ce be para fazer o seu trabalho? & 0,960 & \\
\hline \multirow[t]{3}{*}{ Ouevfut } & Recompensas & $\begin{array}{l}\text { 1. Quão favoráveis serào os beneficios financeiros em longo prazo se você } \\
\text { permanecer na organizacăo? }\end{array}$ & 0,864 & 0,752 \\
\hline & Expectativas Futuras & $\begin{array}{l}\text { 2. Quão favoráveis serão suas oportuniđades de promoção se você permenacer na } \\
\text { organização? }\end{array}$ & 0,877 & \\
\hline & & 3. Qual a segurança de wocê permancer no seu emprego? & 0,700 & \\
\hline \multirow[t]{7}{*}{ Ouevfai } & Recompensas & 2. Eu recebo as oportunidades que mereço no meu trabalho & 0,869 & 0,939 \\
\hline & Econômicas - & 3. A remuneração que recebo está ligada a quão bem realizo meu trabalho. & 0,844 & \\
\hline & Oportunidades Justas & 4. Minha organi zação se preocupa em me remunerar de forma justa. & 0,870 & \\
\hline & & 5. Minha organização quer que eu re ceba o salário e os beneficios que mereço. & 0,887 & \\
\hline & & $\begin{array}{l}\text { 6. Em geral, a remuneração é distribuida de forma justa entre os funcionários de } \\
\text { minha organização. }\end{array}$ & 0,867 & \\
\hline & & 7. Na minha organização os funcionários recebem as oportunidades que merecem. & 0,750 & \\
\hline & & $\begin{array}{l}\text { 8. A remuneração que as pesso as recebem está vinculada a quão bem realizam o seu } \\
\text { trabalho. }\end{array}$ & 0,818 & \\
\hline \multirow[t]{7}{*}{ Exrobe } & Comportamento & Com qual frequência você: & & 0,851 \\
\hline & Discricionário & $\begin{array}{l}\text { 1. Se oferece voluntariamente para fazer atividades extras, a fim de ajudar a } \\
\text { organização? }\end{array}$ & 0,666 & \\
\hline & & 2. Se oferece voluntari amente para ajudar na orientação de novos funcionários? & 0,748 & \\
\hline & & 3. Ajuda colegas a resolver problemas relacionados ao trabalho? & 0,837 & \\
\hline & & $\begin{array}{l}\text { 4. Se oferece para ajudar colegas a fazer o trabalho deles quando eles estão } \\
\text { sobrecarregados? }\end{array}$ & 0,807 & \\
\hline & & $\begin{array}{l}\text { 5. Coloca um esforço extra para ajudar seus colegas a realizar trabalho acima do } \\
\text { esperado? }\end{array}$ & 0,798 & \\
\hline & & $\begin{array}{l}\text { 6. Compartil ha seus conhecimentos com colegas de trabalho, mesmo quando nào } \\
\text { receba reconhecimento por isso? }\end{array}$ & 0,748 & \\
\hline
\end{tabular}

Fonte: Elaborada pelos autores.

Devido ao baixo valor de carga fatorial, foram removidas uma afirmação do constructo Exrobe, uma afirmação do constructo Ouevfai e uma afirmação do constructo ProJus. As variáveis resultantes da análise fatorial apresentaram níveis adequados de confiabilidade, com alfas de Cronbach superiores a 0,70 (HAIR et al., 2009).

Quanto às medidas de adequação da análise fatorial, o teste KMO (Kaiser-Meyer-Olkin) resultou em um valor de 0,943, considerado adequado. $\mathrm{O}$ teste Bartlett de esfericidade se mostrou significante (Chiquadrado $=10397,202 ; \mathrm{df}=1176 ; p<0,001)$, indicando que a análise fatorial é adequada (HAIRet al., 2009).

Como a hipótese do trabalho é verificar a mediação da identidade social na relação entre procedimentos justos e recompensas econômicas com comportamentos discricionários, foram testados juntamente com 
o modelo proposto por Blader e Tyler (2009) (modelo 1), mais duas alternativas: uma delas considerando somente a existência da mediação (modelo 2) e outra sem mediação (modelo 3), conforme mostrado na Figura 2.

Os três modelos estruturais foram testados e ajustados por meio do uso da técnica de equações estruturais, e seus respectivos indicadores de fit se encontram na tabela 5. As correlações entre itens de mesmo constructo foram incorporadas ao modelo, melhorando esses índices, não comprometendo as análises realizadas (BYRNE, 2009).

Figura 2: Comparação de modelos conceituais

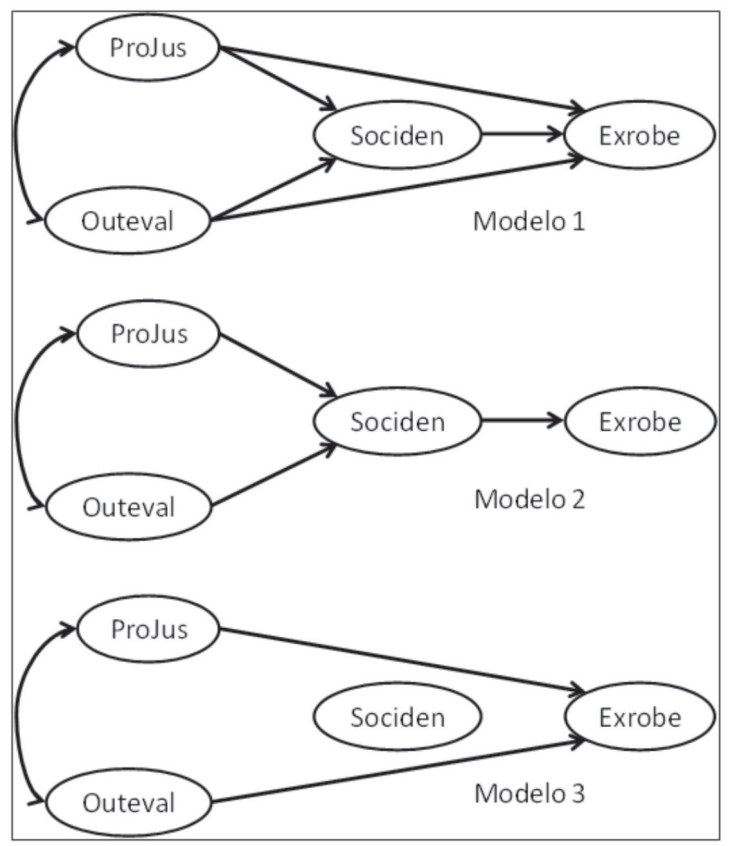

Fonte: elaborado pelos autores 
Tabela 5: Indicadores de fit

\begin{tabular}{|c|c|c|c|c|}
\hline \multirow[t]{2}{*}{ Teste de FIT } & \multirow[t]{2}{*}{ Valor Crítico } & \multicolumn{3}{|c|}{ Obtidos } \\
\hline & & Mod. 01 & Mod. 02 & Mod. 03 \\
\hline $\mathrm{N}$ & & 221 & 221 & 221 \\
\hline Minimum Fit Function Chi-Square & & $1815,917(p=0,000)$ & $1819,558(p=0,000)$ & $2049,768(p=0,000)$ \\
\hline Degree of Freedom & & 951 & 953 & 954 \\
\hline $\mathrm{x} 2 / \mathrm{df}$ & & 1,91 & 1,91 & 2,15 \\
\hline $\begin{array}{l}\text { Root Mean Square Error of Aproximation } \\
\text { (RMSEA) }\end{array}$ & RMSEA $<0,10$ & 0,064 & 0,064 & 0,072 \\
\hline Comparative Fit Index (CFI) & $\mathrm{CFI}>0,9$ & 0,906 & 0,906 & 0,881 \\
\hline Incremental Index of Fit (IFI) & $|F|>0,9$ & 0,907 & 0,907 & 0,882 \\
\hline Tucker-Lewis Index (TLI) & $T L I>0,9$ & 0,898 & 0,898 & 0,871 \\
\hline Parsimony CFI (PCFI) & $\mathrm{PCFI}>0,8$ & 0,832 & 0,834 & 0,812 \\
\hline Resultados & & Aceito & Aceito & Rejeitado \\
\hline
\end{tabular}

Fonte: Elaborada pelos autores.

Como pode ser verificado, o modelo 3 , que não considera a mediação, foi rejeitado por apresentar índices não adequados aos requisitos mínimos (BYRNE, 2009; MARÔCO, 2014). Por outro lado, os modelos 1 e 2 apresentaram-se adequados, sem distinção significante entre eles, quando comparados por meio da relação $\chi^{2} / \mathrm{df}$ (MARÔCO, 2014). Ambas as situações indicam a possível existência da mediação da identidade social.

Seguindo com a avaliação dos modelos 1 e 2, foi realizada a análise da significância das relações entre os construtos, por meio dos coeficientes de regressão. A tabela 6 apresenta tais relações e coeficientes para cada um dos modelos.

A despeito da conclusão anterior sobre o modelo 3, foram apresentados os seus coeficientes e, como esperado, os mesmos não são significantes. O modelo 1 apresenta as relações ProJus/Exrobe e Outeval/Exrobe não significantes, justificando sua similaridade com o modelo 2, que não possui essas relações.

Entre os modelos 1 e 2, o modelo 2 é o mais parcimonioso (BYRNE, 2009), o que, acrescido ao fato das relações acima discutidas não serem significantes, leva à conclusão de que a identidade social efetivamente é mediadora entre procedimentos justos e recompensas econômicas com comportamento discricionário, confirmando a hipótese inicial dessa pesquisa. 
Tabela 6: Coeficientes de regressão

\begin{tabular}{|c|c|c|c|c|}
\hline \multirow[t]{2}{*}{ Modelo } & \multirow[t]{2}{*}{ Relações } & \multicolumn{2}{|c|}{ Coeficientes } & \multirow[t]{2}{*}{ p-valor } \\
\hline & & b & $\beta$ & \\
\hline \multirow[t]{5}{*}{01} & ProJus $\rightarrow$ Sociden & 0,283 & 0,415 & 0,001 \\
\hline & Outeval $\rightarrow$ Sociden & 0,349 & 0,461 & 0,000 \\
\hline & Sociden $\rightarrow$ Exrobe & 0,670 & 0,636 & 0,000 \\
\hline & ProJus $\rightarrow$ Exrobe & $-0,038$ & $-0,052$ & 0,800 \\
\hline & Outeval $\rightarrow$ Exrobe & $-0,204$ & $-0,255$ & 0,235 \\
\hline \multirow[t]{5}{*}{02} & ProJus $->$ Sociden & 0,280 & 0,409 & 0,002 \\
\hline & Outeval $>>$ Sociden & 0,351 & 0,463 & 0,000 \\
\hline & Sociden $\rightarrow$ Exrobe & 0,373 & 0,356 & 0,000 \\
\hline & ProJus $\rightarrow$ Exrobe & - & - & - \\
\hline & Outeval $\rightarrow$ Exrobe & - & - & - \\
\hline \multirow[t]{5}{*}{03} & ProJus $\rightarrow$ Sociden & - & - & - \\
\hline & Outeval $>>$ Sociden & - & - & - \\
\hline & Sociden $\rightarrow$ Exrobe & - & - & - \\
\hline & ProJus $\rightarrow$ Exrobe & 0,173 & 0,247 & 0,192 \\
\hline & Outeval -> Exrobe & $-0,005$ & $-0,007$ & 0,972 \\
\hline
\end{tabular}

Fonte: Elaborada pelos autores.

Tabela 7: Coeficientes de determinação

\begin{tabular}{lccc}
\hline Constructo & \multicolumn{3}{c}{$\mathbf{R}^{2}$} \\
\cline { 2 - 4 } & Modelo 01 & Modelo 02 & Modelo 03 \\
\hline Sociden & 0,730 & 0,725 & - \\
Exrobe & 0,170 & 0,127 & 0,058 \\
\hline
\end{tabular}

Fonte: Elaborada pelos autores.

Complementarmente, assumindo o modelo 2 como aquele com maior capacidade de explicação do fenômeno estudado, a análise do coeficiente de determinação $\left(R^{2}\right)$, conforme apresentado na tabela 7 , mostra que procedimentos justos e recompensas econômicas explicam mais de $70 \%$ da identidade social desenvolvida pelo funcionário da empresa (HAIR et al., 2009). Em compensação, a identidade social, sozinha, explica pouco mais de $10 \%$ do comportamento discricionário do funcionário, o que leva à conclusão de que devem existir outros fatores não previstos no modelo que explicam tal comportamento. 


\section{Conclusão}

O presente trabalho, baseado no estudo de Blader e Tyler (2009) teve como objetivo identificar o papel de mediação da identidade social entre as variáveis preditoras (procedimentos justos e recompensas econômicas) e seus efeitos sobre o comportamento discricionário de funcionários, por meio do modelo de equações estruturais.

Foram testados modelos alternativos, a saber: o modelo completo, envolvendo todas as possíveis relações (modelo 1), e as alternativas com e sem mediação da identidade social(respectivamente modelos 2 e 3).

O modelo 2 assume que a identidade social, que recebe influência direta de procedimentos justos e recompensas econômicas, explica e influencia o comportamento discricionário melhor do que os modelos 1 e 3. Portanto, essa variável age como interveniente entre as variáveis independentes (procedimentos justos e recompensas econômicas) e a variável dependente (comportamento discricionário). De acordo com essas revelações, que são fruto da percepção de funcionários, observa-seque a empresa que oferece remuneração competitiva e adota procedimentos justos para seus funcionários não provoca diretamente neles o comportamento discricionário. Para que tal comportamento aconteça, é necessário que seja desenvolvida a identidade social na organização.

A identidade social não ocorre em curto prazo, mas se cristaliza ao longo do tempo (DECHAMPS; MOLINER, 2009) em decorrência das categorizações desenvolvidas pela organização e que, conforme proposto por Tajfel(1982), formam a identidade do grupo. De acordo a pesquisa realizada no presente trabalho, os procedimentos justos e as recompensas econômicas somente provocarão comportamento discricionário à medida que a identidade social for sendo desenvolvida. Por outro lado, a identidade social somente se reforça na empresa, caso essa insista nas suas políticas relacionadas a procedimentos justos e recompensas econômicas (DECHAMPS; MOLINER, 2009). 
Ficou evidenciado que os procedimentos justos, assim como as recompensas econômicas, desenvolvem o senso de coletividade que contribui para a formação da identidade social e tem como consequência o comportamento discricionário, o que parece corroborar Birnberg et al. (2008), já que tais autores associam o comportamento discricionário a esse senso de coletividade.

Como limitação do estudo, apresenta-se a pouca idade da maioria dos respondentes, com 62,9\% deles entre 17 e 25 anos. De acordo com Leea et al. (2011), funcionários com idade mais baixa em início de carreira, são mais susceptíveis às políticas motivacionais no que se refere à remuneração do que funcionários seniores, o que poderia caracterizar uma tendência nos padrões de resposta. Dessa forma, abre-se a perspectiva de futuras pesquisas que deem continuidade no desenvolvimento dessa temática.

\section{Referências}

ADAMS, J. S. Inequity in social exchange. In: BERKOWITZ, L. Advances in experimental social psychology. New York: Academic Press, v. 2, p. 267-299, 1965.

ASSMAR, E. M. L.; FERREIRA, M. C.; SOUTO, S. O. Justiça organizacional: uma revisão crítica da literatura. Psicologia: Reflexão e Crítica, Porto Alegre, v. 18, n. 3, p. 443-453, Sept./Dec., 2005.

BAKKER, A. B.; ALBRECHT, S. L.; LEITER, M. P. Key questions regarding work engagement. European Journal of Work and Organizational Psychology, [S.I.], v. 20, issue 1, p. 4-28, 2011.

BAKKER, A. B.; BAL, P. M. Weekly work engagement and performance: A study among starting teachers. Journal of Occupational and Organizational Psychology, [S.I.], v. 83, issue 1, p. 189-296, Mar., 2010.

BARON R.M.; KENNY, D.A.. The Moderator-Mediator Variable Distinction in Social Psychological Research: Conceptual, Strategic, 
and Statistical Considerations. Journal of Personality and Social Psychology, Washington, v.51, n.6, p.1173-1182, 1986.

BIRNBERG, J. G.; LUFT, J.; SHIELDS, M. D. Psychology theory in management accounting research. In: CHAPMAN, C. S.; HOPWOOD, A. G.; SHIELDS, M. D. Handbook of Management Accounting Research. [S.I]: Elsevier, 2008.

BLADER, S. L.; TYLER, T. R. Testing and extending the group engagement model: linkages between social identity, procedural justice, economic outcomes, and extra role behavior. Journal of Applied Psychology, College Park, MD v. 94, n. 2, p. 445-464, 2009.

BLAU, P. Exchange and power in social life. New York: Wiley, 1964. BYRNE, B. M. Structural equation modeling with LISREL, PRELIS, and SIMPLIS: Basic concepts, applications, and programming. New York: Psychology Press, 2009.

DECHAMPS, J. C.; MOLINER, P. A identidade em psicologia social: dos processos identitários às representações sociais. Petrópolis: Vozes, 2009.

DEJOURS, C. Uma nova visão do sofrimento humano nas organizações. In: CHANLAT, J. F. O indivíduo na organização: dimensões esquecidas. São Paulo: Atlas, v. 1, p. 149 -174, 1996.

DUBINSKY, A. J.; SKINNER, S. J. Going the extra mile: Antecedents of salespeople's discretionary effort. Industrial Marketing Management, [S.I.], v. 31, n. 7, p. 589-598, 2002.

GINTIS, H.; BOWLES, S.; BOYD, R.; FEHR, E. Moral sentiments and material interests: The foundations of cooperation in economic life. Cambridge, MA: MIT Press, 2005.

HAIR, J. F.; BLACK, W. C.; BABIN, B. J.; ANDERSON, R. E.; TATHAM, R. L. Análise Multivariada de Dados. Porto Alegre: Bookman, 2009. 
HASLAM, S. A. Psychology in organizations: The social identity approach. London: Sage, 2004.

HASLAM, S. A.; ELLEMERS, N. Social identity in industrial and organizational psychology: Concepts, controversies and contributions. In: HODGKINSON, G. P. International review of industrial and organizational psychology. [S.I.], [s.n.], v. 20, p. 39-118, 2005.

HAWORTH, C. L.; LEVY, P. E. The importance of instrumentality beliefs in the prediction of organizational citizenship behaviors. Journal of Vocational Behavior, [S.I.], v. 59, p. 64-75, Aug, 2001.

HOMANS, G. Social behavior: Its elementary forms. New York: Harcourt, Brace, World, 1961.

HOWARD, L. W.; DOGHERTY, T. W. Alternative Reward Strategies and Employee Reactions. Compensation \& Benefits Review, [S.I.], v. 36, n. 1, p. 41-51, Jan./Feb., 2004.

KATARIA, A.; GARG, P.; RASTOGI, R. Employee Engagement and Organizational effectivess: The role of organizational citizenship behavior. International Journal of Business Insights \& Transformation, Navi Mumbai, India, v. 6, n. 1, p. 102-113, Mar., 2013. KENNEDY, E.; DAIM, T. U. A strategy to assist management in workforce engagement and employee retention in the high tech engineering environment. Evaluation and Program Planning, [S.I.], v. 33, n. 4, p. 468-476, Nov., 2010.

KONOVSKY, M. A.; PUGH, S. D. Citizenship behavior and social exchange. Academy of Management Journal, [S.I.], v. 37, n. 3 p. 656669, June, 1994.

LEEA, H. J.; IIJIMAB, Y.; READEC, C. Employee preference for performance-related pay: predictors and consequences for organizational citizenship behavior in a Japanese firm. The International Journal of Human Resource Management, [S.I.], v. 22, n. 10, p. 2086-2109, Mar, 2011. 
MARÔCO, J. Análise de Equações Estruturais: Fundamentos teóricos, software e aplicações. 2a. ed. [S.I.]: ReportNumber, 2014.

NETEMEYER, R. G.; BOLES, R. G.; MCKEE, J. S.; MCMURRIAN, $R$. An investigation into the antecedents of organizational citizenship behaviors in a personal selling context. Journal of Marketing, Chicago, v. 61, n. 3, p. 85-98, Jul,1997.

PODSAKOFF, P. M.; MACKENZIE, S. B.; PAINE, J. B.; BACHRACH, D. G. Organizational citizenship behaviors: A critical review of the theoretical and empirical literature and suggestions for future research. Journal of Management, New York, v. 26, p. 513-563, June, 2000.

TAJFEL, H. Social psychology of intergroup relations. Annual Review of Psychology, v. 33, p. 1-39, Feb.1982.

THIBAUT, J.; KELLEY, H. H. The social psychology of groups. New York: Wiley, 1959.

TYLER, T. R. Social justice: Outcome and procedure. International Journal of Psychology, [S.I.], v. 35, n. 2, p. 117-125, Apr., 2000.

TYLER, T. R.; BLADER, S. L. Cooperation in groups: Procedural justice, social identity, and behavioral engagement. Philadelphia: Psychology Press, 2000.

TYLER, T. R.; BLADER, S. L. The group engagement model: Procedural justice, social identity, and cooperative behavior. Personality and Social Psychology Review, [S.I.], v. 7, n.4, p. 349361, 2003.

VAN DIJKE, M.; WILDSCHUT, T.; LEUNISSEN, J. M.; SEDIKIDES, C. Nostalgia buffers the negative impact of low procedural justice on cooperation. Organizational Behavior and Human Decision Processes, v. 127, p. 15-29, Mar.2015.

VASCONCELOS, I. F. F. G.; VASCONCELOS, F. C. Gestão de Recursos Humanos e Identidade Social: Um Estudo Crítico. RAE - 
Revista de Administração de Empresas, São Paulo, v. 42, n. 1, jan.mar. 2002.

VIEIRA, K. M.; DALMORO, M.. Dilemas na construção de escalas tipo likert: o número de itens e a disposição influenciam nos resultados. In: ENCONTRO DAASSOCIAÇÃO NACIONAL DE PÓS-GRADUAÇÃO EM ADMINISTRAÇÃO, 32, 2008, Anais...Rio de Janeiro: ANPAD, 2008.1 CD ROM

Artigo recebido em: 16/10/2015

Aprovado em: 21/01/2016 